\title{
An Artificial Neural Network For Hedging Crude Oil
}

Hossein Najafi, (E-mail: hossien.najafi@uwrf.edu), University of Wisconsin, River Falls Reza Rahgozar, (E-mail: reza.rahgozar@uwrf.edu), University of Wisconsin, River Falls Brian Champlin, Aerosim Technologies, Inc., Burnsville, MN

\begin{abstract}
A neural network model for hedging crude oil is introduced. The NYMEX futures prices is used to investigate the effectiveness of this model. Empirical results show that the neural network model reduces price risk more than other approaches.
\end{abstract}

\section{INTRODUCTION}

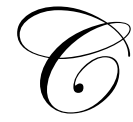

rude oil prices have been volatile over the past several years. Such volatility has created risk that requires active management. To manage risk, various minimum-variance hedging models have been developed (Mahul (2002), Routledge, Sppi, and Spatt (2000), Pirrong (1997), Newbery (1988), and McKinnon (1967)). The minimum-variance models attempt to minimize the variance of a firm's payoffs by selecting the optimal unit of commodity to hedge. Recent empirical study by Rahgozar and Najafi (2003a) shows that although minimum-variance models effectively manage price risk they have a negative effect on revenue. In another study, Rahgozar and Najafi (2003b) have shown that employing an ad-hock hedging strategy can reduce risk without having a strong negative effect on revenue.

Over the past two decades, several learning networks have been developed for modeling nonlinear statistical relationships. In particular, Projection Pursuit Regression (Friedman and Stuetzle (1981)), Multiplayer Perception (Rumelhart (1986), Werbos (1974)), Radial Basis Functions (Poggio and Girosi (1990)), and Support Vector Machine (Vapnik et. al. (1992)) are examples of such techniques. Many researchers (Hutchinson, Andrew and Poggio (1994), Gençay and Qi (2001), Carelli et al (2000))) have used these techniques for pricing and hedging derivative securities as alternatives to the Black and Scholes (1973) and Merton (1973) models.

To manage crude price risk, this article introduces a neural network hedging model. In particular, a feedforward neural networks using the standard back-propagation algorithm (Werbos (1974), Rumelhart (1986), Hagan (1994)) is employed to generate the hedge ratios. Performance of the proposed neural network hedging model and its effectiveness in managing risk is compared with other hedging models. Empirical results show that the neural network approach not only reduces risk but it also has a positive effect on revenue.

The remainder of this article is organized as follows: Section II describes the neural network hedging model used in this study; section III provides performance results of the model in the crude oil market; section IV offers concluding comments and evaluations.

\section{THE NEURAL NETWORK HEDGING MODEL}

To develop the neural network hedging model, a similar approach recommended by Kaastra and Boys (1995) is followed and its steps are depicted in Figure 1 and described below.

The first step in developing such a neural network is to identify appropriate input and output parameters. Generally, historical spot and futures prices and production levels provide predictive power and are considered as input variables. Other parameters that can provide additional significant predictive power, such as CPI and interest rates, are also identified and considered as input variables. 
Figure 1

Steps Involved in Producing the Neural Network Hedging Model

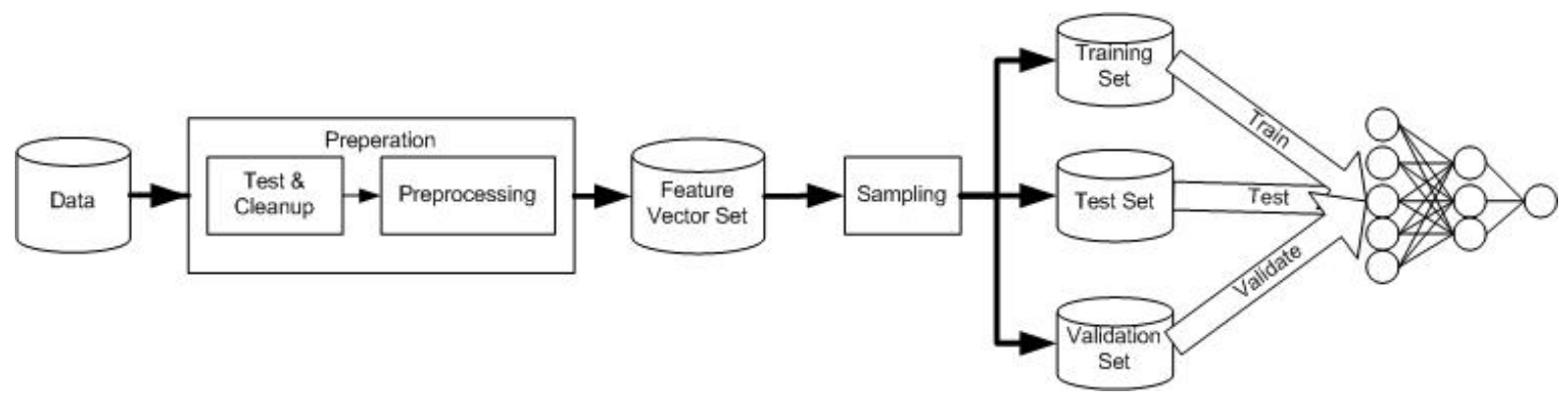

Once the input and output parameters are identified, a historical data set is composed and processed to generate the training, test, and validation sets. These sets are made of feature vectors, each representing one day of the historical data. Each feature vector is composed of a set of input values and a single output value.

To improve performance, deviation from short, medium, and long term norms, instead of the actual variable values were used as inputs to the network (Kaastra and Boys (1995). To accomplish this, the short, medium, and long term z-scores of the corresponding variables were measured and employed.

The target values for the output parameter (i.e. the hedge ratio) were computed based on future performance of the crude oil. It is assumed that during the training phase information regarding the future performance of the crude oil is available and is used to generate the desired hedge ratios. More specifically, for a given day, the futures prices on that day $\boldsymbol{F}_{\boldsymbol{1}}, \boldsymbol{F}_{2}, \ldots, \boldsymbol{F}_{\boldsymbol{n}}$ and the corresponding future spot prices $\boldsymbol{F s}_{1}, \boldsymbol{F s}_{2}, \ldots, \boldsymbol{F s}_{\boldsymbol{n}}$ are used to generate the target hedge ratios. Here $\boldsymbol{F} s_{i}$ is the spot price on the maturity date of the futures $\boldsymbol{F}_{i}$.

For a give day $\mathbf{i}$, the target hedge rations, $\mathbf{h}_{\tau}(\boldsymbol{i})$, is computed as the weighted sum of a revenue maximizing hedge ratio $\boldsymbol{h}_{\alpha}(\boldsymbol{i})$ term and a risk minimizing hedge ratio $\boldsymbol{h}_{\boldsymbol{\beta}}(\boldsymbol{i})$ term.

$h_{\tau}(i)=(1-\gamma) * h_{\alpha}(i)+\gamma * h_{\beta}(i)$

Here, $\gamma$ is between 0 and 1 and it allows for flexibility in adapting the model for the hedgers' need. Note that a $\gamma$ of 1 will lead to hedge ratios that optimize risk, while $\gamma$ of 0 will lead to hedge ratios that optimize revenue.

The revenue maximizing ratio, $\boldsymbol{h}_{\boldsymbol{\alpha}}(\boldsymbol{i})$, is computed based on the relationship between $\boldsymbol{F}_{\boldsymbol{i}}$ and $\boldsymbol{F s}_{\boldsymbol{i}}$. The system recommends hedging if the futures price is higher than the spot price on the maturity date of the futures. That is

$h_{a}(i)=\left\{\begin{array}{cc}1 & \text { if } \\ 0 & \text { otherwise }\end{array} \quad F_{i} \geq F s_{i}\right.$

The risk minimizing ratio, $\boldsymbol{h}_{\boldsymbol{\beta}}(\boldsymbol{i})$, is computed to keep that day's hedged revenue $\boldsymbol{h} \boldsymbol{r}_{\boldsymbol{i}}$ as close as possible to its longterm norm ${ }^{1}$. That is

$h r_{i}=\overline{h r}$ where

\footnotetext{
${ }^{1}$ This reflects the concept of mean reversion that has been considered important in deciding futures prices (E. Schwartz, 1997, Gibson and Schwartz 1990, Ross 1997).
} 
$\overline{h r}=\frac{\sum_{j=1}^{j=n} h r_{i-j}}{n}$ and

$h r_{i}=h_{\beta}(i) * F_{i}+\left(1-h_{\beta}(i)\right) * F S_{i}$

Limiting the hedge ratio to values between 0 and 1 leads to a risk minimizing factor of

$$
h_{\beta}(i)=\left\{\begin{array}{cll}
\left(\overline{h r}-F S_{i}\right) /\left(F_{i}-F s_{i}\right) & & \\
0 & \text { if } & \left(\overline{h r}-F S_{i}\right) /\left(F_{i}-F s_{i}\right)<0 \\
1 & \text { if } & \left(\overline{h r}-F S_{i}\right) /\left(F_{i}-F s_{i}\right)>1
\end{array}\right.
$$

Once the feature vector sets are generated, they are sampled to produce the training, test and validation sets. The most recent contiguous set of observations are selected and used for the validation set. The remaining feature vectors are then randomly sampled to produce the training and test set.

The training set is the largest set, about 60 percent of the complete set, and is used to train the network to predict the target hedge ratios. The test set is about 20 percent of the complete set and is used to evaluate the generalization ability of the network. The validation set is also about 20 percent of the complete set and is used for the final check on the performance of the trained network.

\section{MODEL PERFORMANCE}

The performance of the designed neural network hedging model was extensively tested using crude oil prices. Data used in this study included daily observations of spot prices of WTI (Western Texas Intermediate, Cushing Oklahoma FOB), U.S. crude oil production, and settlement futures prices of NYMEX over the $1990-2002$ periods. In addition, daily North American average temperature, Consumer Price Index (CPI), and the interest rates ${ }^{2}$ were used as inputs to the network. Data was first tested, cleaned up, preprocessed, and then the resulting feature vectors where used to produce the train, test, and validation sets.

One hundred networks were trained and tested and the average performance of the trained networks in terms of risk and revenue management was studied. The best performing network was then selected and its performance was tested using the validation set. (Steps are depicted in Figure 1.)

To investigate the hedging effectiveness of the networks, their average effect on revenue and risk was measured on the test set. Hedging effectiveness of each network was measured by calculating change in unitrevenue (i.e., price) and risk relative to an unhedged approach. Change in unit-revenue, $\Delta \mathbf{r}$, and change in risk, $\Delta \mathbf{s}$, were measured using the following formula.

$$
\Delta r=1-\overline{y^{o}} / \frac{}{y^{h}} \text { and } \Delta s=1-s d\left(y^{0}\right) / s d\left(y^{h}\right)
$$

where

$\overline{y^{0}}, \overline{y^{h}}, s d\left(y^{0}\right)$ and $s d\left(y^{h}\right)$ are the mean and standard deviation of unhedged and hedged unit-revenue respectively.

\footnotetext{
${ }^{2}$ Interest rates were measured by T-Bill rates.
} 
As shown in table I, recommended average hedge ratios vary for different maturity dates and from year to year. However, on average they tend to be just above 50 percent (see the last column of this table).

The performance of the networks in terms of their effect in reducing risk is investigated. For almost all maturity dates and every year in the study, the recommended hedge ratios lead to risk reduction. Nearby futures contracts generated smaller risk reduction than distant futures. As shown in the last column of the Table I, average risk reduction ranges from 7.5 percent for the first month futures to 57.58 percent for the fourth months futures.

As it appears in the table, the neural network model not only reduced risk but at the same time increased or did not affect the revenue. For example, in 1994, using the nearby futures reduced risk by 10.99 percent and increased revenue by 1.13 percent; the fourth-month futures price reduces risk by 125.66 percent and increased revenue by 5.93 percent. For the entire sample, on the average, fourth-month futures show a reduction in risk of 57.58 percent and an increase in revenue of 2.89 percent. An annual plot of the networks' effectiveness on revenue and risk for the fourth month futures is presented in Figure 2.

Table I

Average Hedge Ratios and Measure of Hedging Effectiveness of Networks Using Futures Prices of Different Maturity Dates

\begin{tabular}{|c|c|c|c|c|c|c|c|c|c|c|c|c|}
\hline & & 1 & 1 & 1992 & 19 & 4 & 1 & 1 & 997 & 8 & 10 & $\begin{array}{r}\text { Average } \\
1990-1999\end{array}$ \\
\hline \multirow{4}{*}{ 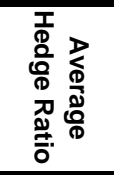 } & F1 & $58.92 \%$ & $54.86 \%$ & $57.52 \%$ & $62.83 \%$ & $45.92 \%$ & $49.47 \%$ & $49.99 \%$ & $49.30 \%$ & $59.51 \%$ & $46.53 \%$ & $53.48 \%$ \\
\hline & $\mathrm{F} 2$ & $62.50 \%$ & $50.72 \%$ & $58.54 \%$ & $73.69 \%$ & $48.04 \%$ & $51.52 \%$ & $51.07 \%$ & $64.92 \%$ & $74.09 \%$ & $46.61 \%$ & $58.17 \%$ \\
\hline & F3 & $59.63 \%$ & $40.03 \%$ & $48.77 \%$ & $83.91 \%$ & $43.56 \%$ & $41.15 \%$ & $50.11 \%$ & $65.70 \%$ & $75.89 \%$ & $42.64 \%$ & $55.14 \%$ \\
\hline & $\mathrm{F4}$ & $58.75 \%$ & $40.03 \%$ & $50.38 \%$ & $84.42 \%$ & $36.73 \%$ & $46.62 \%$ & $50.56 \%$ & $70.83 \%$ & $81.92 \%$ & $43.11 \%$ & 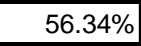 \\
\hline \multirow{4}{*}{ 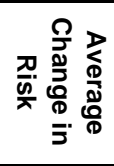 } & F1 & $-9.73 \%$ & $-13.16 \%$ & $-5.07 \%$ & $-2.76 \%$ & $-10.09 \%$ & $-3.68 \%$ & $-11.0<\%$ & $-14.23 \%$ & $-5.23 \%$ & $0.47 \%$ & $-7.45 \%$ \\
\hline & $\mathrm{F} 2$ & $3.58 \%$ & $-30.68 \%$ & $9.68 \%$ & $-3.01 \%$ & $-38.90 \%$ & $30 \%$ & $0 \%$ & $37 \%$ & $76 \%$ & $-4.31 \%$ & $-23.41 \%$ \\
\hline & F3 & $-46.01 \%$ & $-52.05 \%$ & $-41.58 \%$ & $-9.01 \%$ & $-80.71 \%$ & $-13.11 \%$ & $-36.71 \%$ & $-63.58 \%$ & $-17.28 \%$ & $-17.06 \%$ & $-37.71 \%$ \\
\hline & $\mathrm{F4}$ & $-70.53 \%$ & $-81.36 \%$ & $-65.96 \%$ & $-17.15 \%$ & $-125.66 \%$ & $10.06 \%$ & $-49.09 \%$ & $-101.23 \%$ & $-42.68 \%$ & $-32.15 \%$ & $-57.58 \%$ \\
\hline \multirow{4}{*}{ 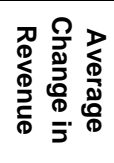 } & F1 & $0.75 \%$ & $-0.50 \%$ & $0.09 \%$ & $32 \%$ & $1.13 \%$ & $0.24 \%$ & $1.38 \%$ & $-0.33 \%$ & $1.09 \%$ & $2.35 \%$ & $0.65 \%$ \\
\hline & $\mathrm{F} 2$ & $2.61 \%$ & $-0.28 \%$ & $0.85 \%$ & $1.37 \%$ & $2.81 \%$ & $0.04 \%$ & $0.36 \%$ & $-0.01 \%$ & $3.09 \%$ & $5.86 \%$ & $1.67 \%$ \\
\hline & F3 & $2.79 \%$ & $-0.28 \%$ & $1.95 \%$ & $1.79 \%$ & $4.67 \%$ & $30 \%$ & $-0.82 \%$ & $-1.18 \%$ & $.53 \%$ & $9.21 \%$ & $2.30 \%$ \\
\hline & $\mathrm{F4}$ & $3.22 \%$ & $-0.55 \%$ & $2.65 \%$ & $2.47 \%$ & $5.93 \%$ & $1.35 \%$ & $-2.41 \%$ & $-1.68 \%$ & $7.19 \%$ & $10.69 \%$ & $2.89^{\circ}$ \\
\hline
\end{tabular}

In comparison with previous hedging models (Rahgozar and Najafi (2003A), Mahul (2002), Routledge, Sppi, and Spatt (2000), Pirrong (1997), Newbery (1988), and McKinnon (1967)), the neural network model performed remarkably well. For example, for the forth month futures, the neural network model reduce risk by 57.58 percent while improving revenue by 2.89 percent. By comparison, the minimum-variance hedging models results tested by Rahgozar and Najafi (2003a), reduced average risk by about 18 percent at a cost of about 2 percent in revenue, while the revenue maximizing models, on average, reduced risk by 4 percent and improved revenue by only about 1 percent.

The last three years of data (2000-2002) was used to further test the performance of the network on an outof-sample validation set. Out of the 100 trained networks, the one with the least mean square error on the test set was selected and used for this purpose. As expected, a performance drop on the validation set was observed. However, in general the networks behavior on the validation set was fairly comparable with its performance on the test set. Hedging effectiveness of the network on the validation set is presented in Table II. Although, there is some performance degradation, the effect of networks hedging on risk is still significant, while its effect on revenue is minimal. 
Figure 2

Plot of Networks' Effectiveness on Revenue and Risk for the Fourth Month Futures

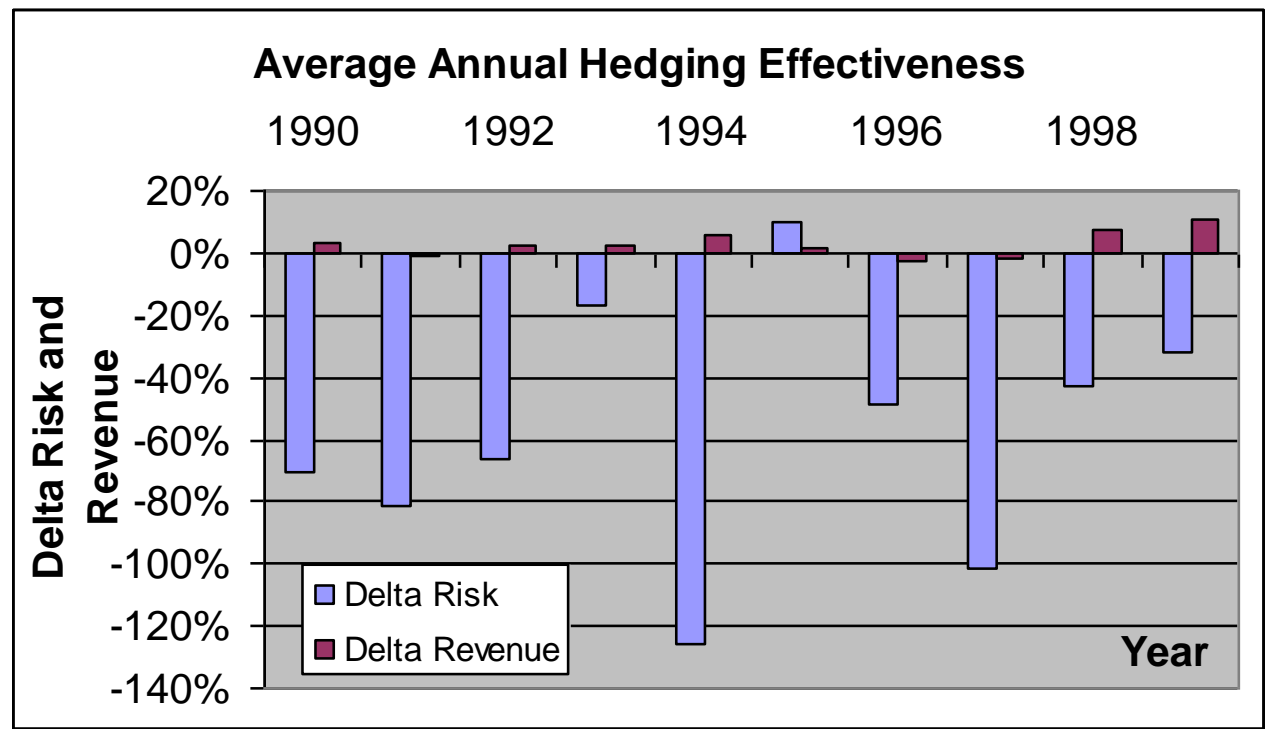

Delta Revenue, $\Delta \mathbf{r}$, and Delta Risk, $\Delta \mathbf{s}$, are measured as $\Delta r=1-\overline{y^{o}} / \overline{y^{h}}$ and $\Delta s=1-s d\left(y^{0}\right) / s d\left(y^{h}\right)$ where $\overline{y^{0}}, \overline{y^{h}}, s d\left(y^{0}\right)$ and $s d\left(y^{h}\right)$ are the mean and standard deviation of unhedged and hedged unit-revenue respectively.

Table II

Average Hedge Ratios and Measure of Hedging Effectiveness of the Best Networks Using Futures Prices with Different Maturity Dates

\begin{tabular}{|c|c|c|c|c|c|}
\hline & & 2000 & 2001 & 2002 & $\begin{array}{r}\text { Average } \\
2000-2003\end{array}$ \\
\hline \multirow{4}{*}{ 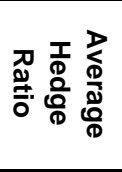 } & F1 & $53.62 \%$ & $49.37 \%$ & $68.87 \%$ & $57.29 \%$ \\
\hline & F2 & $69.84 \%$ & $66.58 \%$ & $58.03 \%$ & $64.82 \%$ \\
\hline & F3 & $43.19 \%$ & $61.14 \%$ & $43.27 \%$ & $49.20 \%$ \\
\hline & F4 & $85.70 \%$ & $78.54 \%$ & $47.51 \%$ & $70.58 \%$ \\
\hline \multirow{4}{*}{ 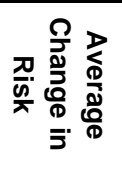 } & F1 & $-7.53 \%$ & $-2.97 \%$ & $4.51 \%$ & $-2.00 \%$ \\
\hline & $\mathrm{F} 2$ & $-11.77 \%$ & $-6.20 \%$ & $-4.60 \%$ & $-7.53 \%$ \\
\hline & F3 & $-20.03 \%$ & $-9.37 \%$ & $-10.49 \%$ & $-13.30 \%$ \\
\hline & F4 & $-17.25 \%$ & $-26.46 \%$ & $-74.71 \%$ & $-39.48 \%$ \\
\hline \multirow{4}{*}{ 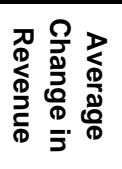 } & F1 & $0.33 \%$ & $-1.22 \%$ & $1.10 \%$ & $0.07 \%$ \\
\hline & F2 & $-2.58 \%$ & $-2.01 \%$ & $2.19 \%$ & $-0.80 \%$ \\
\hline & F3 & $-0.54 \%$ & $-4.03 \%$ & $4.83 \%$ & $0.09 \%$ \\
\hline & $\overline{F 4}$ & $-7.36 \%$ & $-2.01 \%$ & $5.84 \%$ & $-1.18 \%$ \\
\hline
\end{tabular}

\section{CONCLUSION}

A new neural network based hedging model was introduced and its performance in the crude oil market was investigated. The NYMEX futures prices with different maturity dates were employed to hedge the WTI spot 
price risk using the proposed model. The empirical results demonstrate that the neural network model not only reduces price risk but also increases or has no effect on revenue.

\section{BIBLIOGRAPHY}

1. Black, F. and Scholes, M. (1973), “The Pricing of Options and Corporate Liabilities”, J. Political Economy, 81, 18, pp. 637-654.

2. Boser, B. E., Guyon I. M. and Vapnik, V. N. (1992), "A Training Algorithm for Optimal Margin Classifiers", 5th Annual ACM Workshop on COLT, D. Haussler, Ed. Pittsburgh, PA: ACM, pp. 144-152.

3. Carelli, A., S. Silani and F. Stella (2000), "Profiling Neural Networks for Option Pricing," International Journal of Theoretical and Applied Finance, Vol. 3, 2, 183-204.

4. Friedman, J. H. and Stuetzle, W. (1981), "Projection Pursuit Regression," Journal of the American Statistical Association, Vol 76, 1981, pp. 817-823.

5. Gençay, R. and Qi M. (2001), "Pricing and Hedging Derivative Securities with Neural Networks: Bayesian Regularization, Early Stopping and Bagging, IEEE Transactions on Neural Networks" (Special Issue on "Neural Networks in Financial Engineering"), 12(4), 726-734.

6. Gibson Rajna, and E. Schwartz (1990), "Stochastic Convenience Yield and Pricing Oil Contingent Claims," The Journal of Finance, 45, 959-976.

7. Hagan, M.T. and Menhaj M. (1994), "Training Feedforward Networks with Marquardt Algorithm," IEEE transactions on Neural Networks, Vol. 5, and No. 6.

8. Hutchinson, J. M., W. L. Andrew. and Poggio T. (1994), "A Nonparametric Approach to Pricing and Hedging Derivative Securities via Learning Networks," Journal of Finance, Vol. 49, no. 3, July 1994, pp 851-889

9. Kaastra, I. and Boyd, M.S. (1995), "Forecasting Futures Trading Volume Using Neural Networks," Journal of Futures Markets, 15(8), December, 953-70.

10. Mahul, Oliver (2002), "Hedging in Futures and Options Markets with Basis Risk," The Journal of Futures Markets, 22:1, 59-72.

11. Maruyama M., Girosi F. and Poggio T. (1991), "A Connection Between GRBF and MLP, Artificial Intelligence" Memo 1291, Massachusetts Institute of Technology.

12. McKinnon, R.I. (1967), "Futures Markets, Buffer Stocks, and Income Stability for Primary Producers," Journal of Political Economy, 75, 844-61.

13. Merton, R. (1973), "Theory of Rational Option Pricing," Bell Journal Economics Management Science, 4 $141-83$.

14. Newbery DMG (1988), "On the Accuracy of the Mean-Variance Approximation for Futures Markets," Economic Letters, 28, 63-68.

15. Pirrong, S.C. (1997), "Metallgesellschaft: A Prudent Hedger Ruined, or a Wildcatter on NYMEX?" The Journal of Futures Markets, 17, 543-578.

16. Poggio, T. and Girosi, F.(1990), "Network for Approximation and Learning", Proceeding of the IEEE 78, 1481-1497.

17. Rahgozar, R., and Najafi H.L. (2003a), "An Empirical Comparison of Hedging Models In Managing Oil Revenue Risk," Journal of the Academy of Finance, Vol 1, 100-113.

18. Rahgozar, R. and Najafi H. L. (2003b), "Effect of Diversification on Managing Revenue and Risk: An Empirical Analysis Using Crude Oil Futures Markets," Journal of Derivatives Use, Trading \& Regulation, Vol 9, No. 2, pp. 133-149.

19. Ross, Stephen A. (1997), "Hedging Long Run Commitments: Exercises in Incomplete Markets Pricing," Economic Notes by Banca Monte, 29, 99-132.

20. Routledge, R. Bryan, D. Seppi, and C. Spatt (2000), "Equilibrium Forward Cures for Commodities," The Journal of Finance, 55, 1297-1338.

21. Rumelhart, D.E., Hinton G.E. and Williams R.J. (1986), "Learning Internal Representation by Error Propagation," Parallel Distributed Processing, Chapter 8, MIT Press.

22. Schwartz, S. Eduardo (1997), "The Stochastic Behavior of Commodity Prices: Implication for Valuation and Hedging," The Journal of Finance, 52, 923-973.

23. Werbos, P.J. (1974), "Beyond Regression: New Tools for Prediction and Analysis in the Behavioral Sciences," Ph.D. Thesis, Harvard University, Cambridge, MA. 